\title{
Flight Test of Pilot-Aircraft Haptic Feedback System
}

\author{
Pavel Zikmund ${ }^{1,}$, Lukáš Dubnický ${ }^{1}$, Michaela Horpatzká ${ }^{1}$, Miroslav Macík ${ }^{1}$, and Ivo \\ Jebáček $^{1}$ \\ ${ }^{1}$ Brno University of Technology, Faculty of Mechanical Engineering, Technická 2896/2, 616 69, \\ Brno, Czech Republic
}

\begin{abstract}
This research is focused on an innovative improvement of pilotaircraft interaction and is targeted on small aircraft. Haptic feedback is performed by actuators mounted on an aircraft's controls. The purpose of the actuators on a control stick and pedals is stall warning and a pilot guiding to safe and economical flight regimes. The feedback system mediates airflow data as angles of attack and sideslip. The paper brings results of a flight test of the proposed system. Qualitative evaluation of the haptic feedback inflight is presented. Benefits of the system are presented on a sideslip during turning. Some recommendations for the haptic pilotaircraft interaction are stated within the discussion of the flight test results.
\end{abstract}

\section{Introduction}

The control of small aircraft works on the same principle as the control of big airliners, but one aspect is changing recently. Airliners are heading to autonomous flights. Pilots are not required in airlines because of the airplane's physical control but mostly because of the passenger's thrust and safety [1]. The same trend shows the research of Single-PilotOperations [2], which leads to a replacement of a second pilot by advanced onboard automation and/or ground operators providing pilot support services. The situation in small aircraft control is different. Sport and leisure time pilots want to keep control of airplanes. At the same time, a human factor is the most common accident reason which leads to a fatal stall/spin or loss of control accidents. This statistic is stated by EASA and NTBS [3, 4]. Ballistic parachute systems are the best current solution to the loss of control. Company Galaxy recently stated that 95 lives were saved by their system [5]. This research aims to prevent those situations in which rescue systems are required. The improvement of the pilot-airplane interaction is supposed to make the airplane control more intuitive. A decrease in a pilot workload and improvement of a pilot's situational awareness are expected at the same time [6].

Haptic feedback promises such improvement in aircraft control. The survey [6] shows that the transfer of some sensations from visual modality to a touch modality could better utilize the pilot's attention. Beeftink et al. stated [7] that haptic feedback in aircraft control could decrease pilot head-down time on behalf of head-up time on a flight simulator. Active controls providing haptic feedback were designed and tested on a flight simulator [8] in a recent project. Shape morphing and vibrations were compared for control stick guidance [9]. This endeavour leads to a hardware setup with a combination of both functions. The moving element is implemented into the control stick and vibration motors are mounted to the rudder pedals. The vibration pedals application is similar to US patent [10] where

\footnotetext{
* Corresponding author: zikmund@fme.vutbr.cz
} 
pedals indicate sideslip measured by pressure sensors. The active control stick and pedals hardware were tested on a flight simulator at first. After that, a need to verify the function of the hardware in a real aircraft operation appeared. The process of flight test and the results are described in this paper.

The haptic feedback system function and hardware, as well as flight test setup, are described in the following section. The hardware was mounted and tested on ultralight aircraft Dynamic WT-9. A methodology and results of the flight test are stated in the next sections. The discussion section consists of qualitative and quantitative assessment and recommendations for haptic feedback aircraft control applications.

\section{Haptic feedback system}

Haptic feedback system itself consists of sensors, a control unit and haptic elements located in pedals and a control stick handle. Input data are obtained from vanes for the angle of attack (AoA) and angle of sideslip (AoS) measurement and processed by the control unit, which operates the active elements accordingly. AoA is linearly transformed into symmetrical extension and retraction of an active element on the control stick handle. AoS is linearly transformed into asymmetrical extension and retraction of the same active element resulting in tilting movement on the respective side of the airplane sideslip. Both movements can be sensed by the pilot's fingers. When AoS exceeds predefined level, pedal vibration on the respective side is activated. AoS is then negotiated by pressing the vibrating pedal. Pedal vibrations use the pulsing pattern instead of continuous vibrations.

\subsection{Hardware}

The aircraft used for the flight test is a low-wing, single-engine, 2-seats ultralight airplane Dynamic WT-9. A pitot tube with AoA and AoS vanes was used and fixed under the wing of the airplane. The accelerometer [11] was used and fixed inside the cockpit for the flight data evaluation purposes. The control unit was based on an Arduino mega board [12] with data logger shield for flight data recording. The control unit allowed the inflight monitoring of the haptic system using LCD and changing of the haptic feedback system parameters and operation modes. All electronics parts of the control unit were enclosed in $3 \mathrm{D}$ printed (PLA) box. 3D printed (PLA) control stick handle includes two servomotors used for the movement of the active element. The handle was strapped to the airplane's control stick so it would not restrict any of the required movements. Each 3D printed (PLA) rudder pedal extension holds two vibration engines [13].

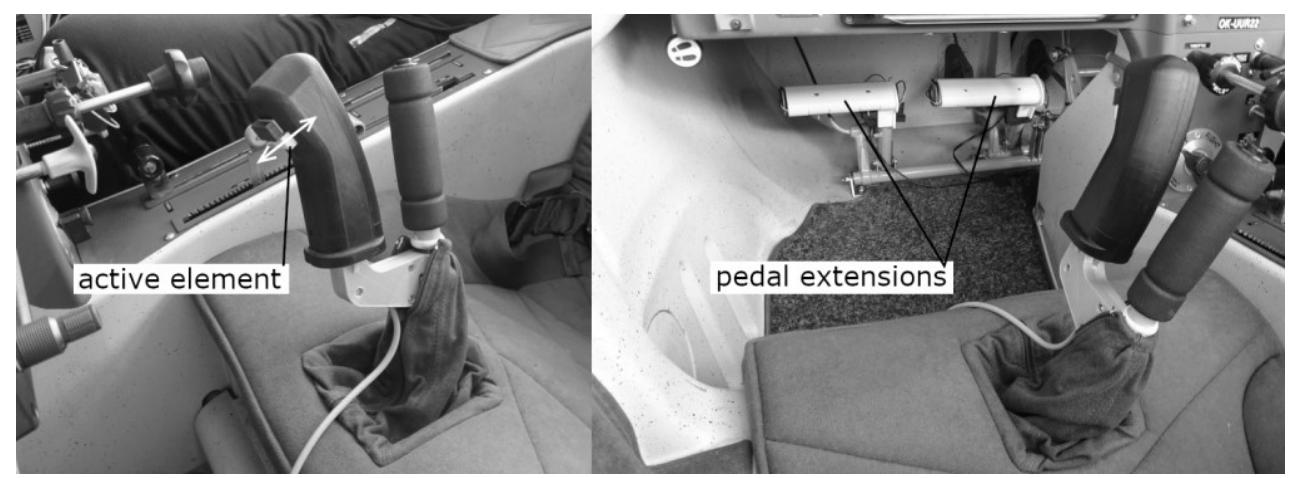

Fig. 1. Haptic feedback actuators on the control stick and the pedals. 
Pedal extensions were fixed to the rudder pedals with several separating layers of microporous rubber to prevent vibration propagation into the aircraft control system.

\section{Methods}

The flight test of the haptic feedback system consisted of the following tasks. The test started by calibration and continued by a subjective assessment, and 360 degrees turns. The goal of the calibration part was to set up reference values of input and output parameters. Zero AoS in symmetric flight was corrected by offset value in horizontal flight. AoA in reference regimes was read and the active element positions were adjusted in required positions. Reference positions of the active element were given by a reference element placed above the active element and by the surface of the control stick handle. The outer reference position marked by the reference element corresponds to AoA at minimal practicable speed. The inner reference position marked by the handle surface corresponds to AoA at the cruise regime speed.

The subjective assessment aimed to inflight verification of the haptic feedback by a pilot. This task was repeated on different speeds within the range between safe near-stall and maximal speed. The pilot commented intensity of rudder pedals vibrations after sideslip flight was induced. There were two issues to be check. The first was possible spreading of vibrations from one pedal to the opposite pedal. The second issue was interference between natural aircraft vibrations induced by the propulsion system and flow field. Positions and function of the active element on the control stick were tested at the same speeds as vibrations of rudder pedals.

The last test task was 360 degrees turns. Left and right turns were performed. One half was flown without the haptic feedback and the second was with the haptic feedback. The haptic feedback was alternately switched on and off to avoid learning effect. AoS was measured as a parameter for the haptic feedback system evaluation. The first hypothesis was: Haptic feedback decreases the mean value of sideslip angle during turning. The second hypothesis was: Haptic feedback decreases sideslip angle above the vibration threshold, which was set up to 5 degrees.

\section{Evaluation}

The haptic feedback system was assessed in horizontal straight flight at first. The range of AoA to be used with the active element position inflight was measured as a part of calibration of the system. The maximal AoA was measured in horizontal flight at minimal practicable airspeed. The AoA for flaps 0, 15, 24 degrees was 19, 18, 18 degrees respectively at airspeeds of $100,85,80 \mathrm{~km} / \mathrm{h}$ respectively. The minimal AoA was 7 degrees measured at cruising speed of $180 \mathrm{~km} / \mathrm{h}$ with flaps retracted. These angles were measured from the estimated horizontal aircraft axis; therefore, the absolute values are rather high. The active element was described by the pilot as well sensible with changes in AoA but with continuous wobbling movement that was rather disturbing.

Onboard gauges were used to achieve flight with zero AoS at cruising speed of 180 $\mathrm{km} / \mathrm{h}$. It was checked that vanes for the haptic feedback system were also reading 0 at that regime. Afterward, the flight with sideslip was induced by the pilot. Vibration haptic feedback activated when onboard sideslip indicator shows half of the ball out of the bracket at the cruise speed. The pilot commented vibrations as well as sensible and sidewise 
unambiguous, even better than on the flight simulator with similar haptic feedback system he tried before. The same sequence was used to check the behaviour of sideslip signalization of the control stick element with tilting movement. Recognizability was assessed as considerably worse than the one of vibrating pedals. Moreover, the pilot said that this feedback could guide him into the roll input with the opposite effect of increasing the sideslip.

Twelve horizontal turns were performed on the indicated airspeed $140 \mathrm{~km} / \mathrm{h}$. The average sideslip was evaluated in the following way. The angle of sideslip range was cut into small intervals. Counting of sideslip angles for each interval gave its distribution. The distribution was normalised by dividing of measured record length. This normalised distribution of sideslip angle was evaluated for each turn separately and for combined groups of turns with and without haptics (Fig. 2). Normalised angle of sideslip was counted by the integration of the distribution by AoS. The resultant normalised sideslip angles were compared by one-tailed t-test. Mean values of normalised sideslip angles in the turns without haptics is $(\mathrm{M}=1.86 \mathrm{deg}, \mathrm{SD}=0.78 \mathrm{deg})$ and in turns with haptics is $(\mathrm{M}=1.66$ $\mathrm{deg}, \mathrm{SD}=0.43 \mathrm{deg})$. It means, that the difference is not statistically significant, $\mathrm{t}(10)=$ $0.54, \mathrm{p}=0.30$. The count of flight time when aircraft sideslip was greater than given threshold 5 deg was also evaluated from normalised distributions of the sideslip. The time when the sideslip was greater than the threshold was $(\mathrm{M}=4.32, \mathrm{SD}=6.06)$ percent of the total time without haptics and $(\mathrm{M}=2.60, \mathrm{SD}=2.84)$ percent of total time with haptics. That means, that haptics decreased sideslip angles above the threshold, but the difference is not statistically significant, $\mathrm{t}(10)=0.63, \mathrm{p}=0.27$.

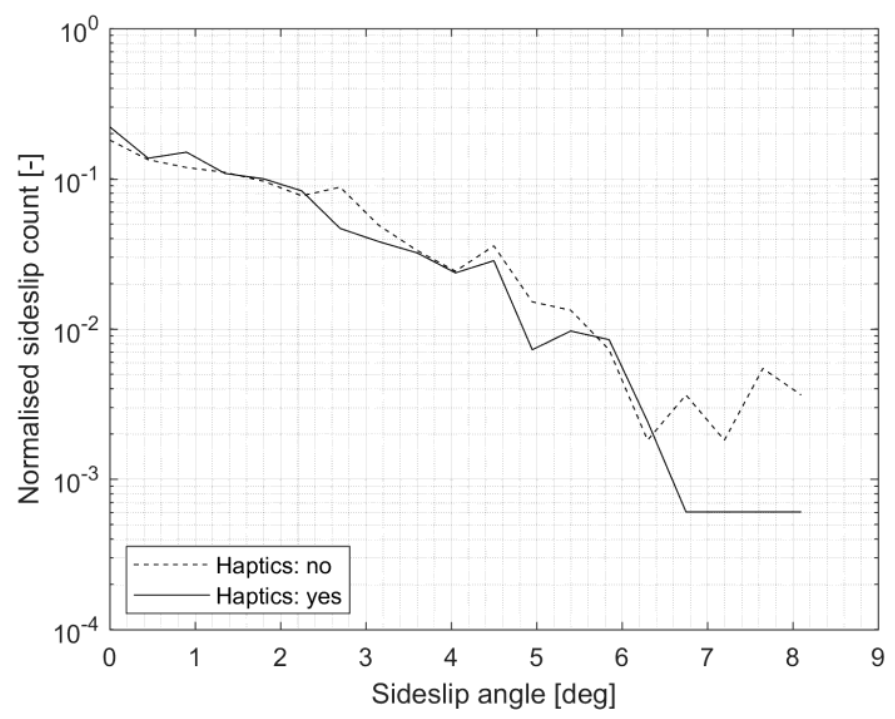

Fig. 2. Normalised sideslip angle distribution with and without haptics.

\section{Discussion}

The active element of the control stick was described by the pilot as well sensible but with disturbing continuous wobbling movement. That movement of the control stick active element was partially caused using insufficient filtering of AoA input in the control unit and by coarse digital conversion, which caused insensitive AoA input. That also limited the 
data for the qualitative assessment of the turns. Changes to filtering and digital conversion are expected to resolve the deficiency in the future.

In the beginning, during past flight simulator tests, some pilots doubted whether vibrations as haptic feedback are suitable for motorized aircraft. We were concerned that vibrations would interfere with the airplane engine vibrations and that vibration of one pedal would spread to the other pedal devaluating the haptic feedback completely. However, vibrations of the haptic pedals were commented by the pilot as well sensible and sidewise unambiguous after the flight test in the whole range of tested speeds. That is an improvement compared to the flight simulator test conducted previously with a similar haptic feedback system. The flight simulator rudder pedals have parallelogram guidance of the pedals with a short mechanical link between the pedals. The airplane used for the flight tests has T-shaped rudder pedals with a longer and less stiff mechanical link between the pedals. That combined with a change in treadles shape and therefore change in pedals attachments housing the vibration motor, lead to the improvement of directional sensibility of rudder pedal vibrations. One of the control stick functions had the same purpose as the vibrating pedals. Tilting movement of the control stick active element was assessed as ambiguous and non-intuitive for sideslip information mediation. The pilot was confused by perceiving both AoA and AoS through the moving element at the same time. Tilting movement of the control stick active element was concluded to be more suitable for a roll instead of yaw guidance. A pilot limb would be perceiving and acting in that case. Roll guidance by the tilting function of the control stick seems to be promising and should be analysed.

Quantitative evaluation of the haptic feedback benefit was tested on the aircraft sideslip during 360 degrees turns. The overall sideslip decreased in case of flight with the haptic feedback, but the improvement was not statistically significant. That could be caused by a small statistical sample. Also, sample turns were all flown by a single pilot. It is expected that the perception and influence of haptic feedback may be strongly personal-related. The test pilot did not complete training of using the haptic feedback system. He only had experience from two-hours flight simulator test, which he participated 5 months before flight test. Figure 2 presents normalised sideslip distribution during twelve 360 degrees turns. There is a visible decrease of sideslip with haptic feedback around sideslip 5 degrees and over 6.3 degrees. It corresponds to the threshold of 5 degrees when the pedals vibrations were activated. It can be supposed that the decrease in the threshold value would help to decrease a sideslip during flight. The threshold level should be decreased only to an appropriate level. A too low value would lead to excessively frequent haptic information that would disturb a pilot during the flight with no further positive effect. The second assumption for the haptic feedback benefit improvement is a pilot training on usage of the haptic feedback system. The system is designed to be intuitive, but ongoing research shows a significant learning effect for this pilot-aircraft interaction.

The last point to be discussed is the system suitability for small aircraft. For the testing purposes, vanes located on the pitot tube were used to measure the AoA and AoS. But this solution is impractical for small aircraft mostly because of its price and vulnerability during ground handling. Supposed solution for a commercially offered system of this kind is expected to include AoA pitot tube that uses only pressure measurements for AoA sensing and lateral accelerometer to substitute the AoS measurements by the acceleration measurements. The same approach with lateral acceleration for sideslip indication is used in widely spread avionics systems like $[14,15]$. 


\section{Conclusions}

The flight test of the haptic feedback system with active element on the control stick and vibration rudder pedals was conducted on the small aircraft. The goal of the flight test was to verify the usability of the haptic feedback previously tested only in a flight simulator. Readability of the active element in the control stick and vibration rudder pedals was assessed positively. Qualitative assessment of the haptic system showed a positive influence on pilot performance in turning flight, but the benefit was not statistically significant. The flight tests revealed some deficiencies that need to be resolved in future development. Complex testing, including pilot training, was recognised as the following step to gain the best profit of the haptic feedback system. In general, the results showed promising potential of the haptic feedback system as an improvement of a pilot-aircraft interaction.

This research was supported by TACR, project no. TJ01000122 "Haptic feedback of assistant systems for flight safety improvement".

\section{Abbreviations}

AoA angle of attack

AoS angle of sideslip

M mean value

PLA polylactic acid

SD standard deviation

\section{References}

1. S. M. Vance, A. S. Malik, Analysis of factors that may be essential in the decision to fly on fully autonomous passenger airliners, J. of Adv. Transport. 49-7, 829-854 (2015)

2. K. D. Bilimoria, W Johnson, P. C. Schutte, Conceptual framework for single pilot operations, Proc. of the International Conf. on HCI in Aerospace, 4 (2014)

3. EASA, Annual Safety Report 2017, European Aviation Safety Agency (2017)

4. NTBS, Most Wanted List 2015, National Transport Safety Board (2015)

5. Galaxy GRS s.r.o., [online] Available at: https://www.galaxysky.cz/?lng=en [Accessed 30 Aug. 2019]

6. P. Zikmund, M. Macík, P. Dvořák, Z. Míkovec, Bio-inspired Aircraft Control, AEAT, 90-6, 983-991 (2018)

7. D. G. Beeftink, et al, Haptic Support for Aircraft Approaches with a Perspective Flight-Path Display, 2018 IEEE I. Conf. on SMC, 3016-3021 (2018)

8. P. Zikmund, M. Macík, Utility model, A system coupled to an aircraft's control lever for tactile communication to maintain a safe flight mode, (2019)

9. P. Zikmund, M. Macík, L. Dubnický, M. Horpatzká, Comparison of Joystick Guidance Methods, Special Issue on CogInfoCom, (to be published) 
10. (J. H. Milgram, U.S. Patent Application No. 10/975,112., (2007) Available at: https://patents.google.com/patent/USH2206H1/en [Accessed 17 Sep. 2019]

11. MPU-6000 and MPU-6050 Product Specification. Rev. 3.4. [ebook] Sunnyvale: InvenSense Inc. Available at: https://www.invensense.com/wpcontent/uploads/2015/02/MPU-6000-Datasheet1.pdf [Accessed 30 Sep. 2019].

12. Store.arduino.cc. Arduino Mega 2560 Rev3. [online] Available at: https://store.arduino.cc/arduino-mega-2560-rev3 [Accessed 30 Aug. 2019]

13. Precisionmicrodrives.com. Product Catalogue - Precision Microdrives, Model No. $307-$ 103 9mm Vibration Motor - 25mm Type. [online] Available at: https://www.precisionmicrodrives.com/product/307-103-9mm-vibration-motor-25mmtype [Accessed 30 Aug. 2019]

14. G5 Electronic Flight Instrument Pilot's Guide for Certified Aircraft. Rev. A. [ebook] Olathe: Garmin Ltd., p.34. Available at: http://static.garmin.com/pumac/190-0111212 A.pdf [Accessed 30 Sep. 2019]

15. SkyView HDX Pilots User Guide. Rev. D. [ebook] Woodinville: Dynon Avionics, sect.4 p.21. Available at:

https://www.dynonavionics.com/includes/guides/SkyView_HDX_Pilots_User_GuideRev_D_v15_4.pdf [Accessed 17 Sep. 2019] 